Annuaire suisse de politique de développement

8 | 1988

Annuaire Suisse - Tiers Monde 1989

\title{
Firmes transnationales : instruments du développement ou instruments de pouvoir?
}

Transnationale Firmen : Faktoren der Entwicklung oder Machtinstrumente?

Gérard Perroulaz

\section{(2) OpenEdition}

1 Journals

Édition électronique

URL : https://journals.openedition.org/aspd/1287

DOI : $10.4000 /$ aspd. 1287

ISSN : 1663-9669

Éditeur

Institut de hautes études internationales et du développement

Édition imprimée

Date de publication : 1 décembre 1988

Pagination : 203-218

ISSN : 1660-5934

\section{Référence électronique}

Gérard Perroulaz, «Firmes transnationales : instruments du développement ou instruments de pouvoir? ", Annuaire suisse de politique de développement [En ligne], 8| 1988, mis en ligne le 13 mars 2013, consulté le 21 septembre 2021. URL : http://journals.openedition.org/aspd/1287 ; DOI : https:// doi.org/10.4000/aspd. 1287 


\section{Firmes transnationales: instruments du développement ou instruments de pouvoir?}

Bibliographie commentée sur les effets des activités des firmes transnationales suisses dans les pays en voie de développement 


\section{Zusammenfassung: Transnationale Firmen: Faktoren der Entwicklung oder Machtinstrumente?}

Bibliographie mit Anmerkungen über die Auswirkungen der schweizerischen transnationalen Firmen in den Entwicklungsländern.

Der vorliegende Beitrag gibt einen Überblick über die Literatur, die sich mit den Auswirkungen der Tätigkeit der schweizerischen transnationalen Firmen in den Entwicklungsländern befasst.

Der erste Teil des Artikels versucht, die wichtigsten Merkmale der Literatur (Auswirkungen der transnationalen Firmen auf Beschäftigung und Ausbildung, Beziehungen zu den Nationalstaaten, usw.) aufzuzeigen und die Literatur in den Rahmen der in den letzten zwanzig Jahren in der Schweiz aufgetretenen Kontroversen zu stellen.

Das Literaturverzeichnis im zweiten Teil umfasst Werke und Artikel aus Fachzeitschriften über die Auswirkungen der transnationalen Unternehmen in den Entwicklungsländern, welche im Zeitraum von 1980 bis Juli1988 erschienen sind. Bei der Auswahl wurden weder die Folgen für die Schweizer Wirtschaft noch Beschreibungen transnationaler Firmen ohne Bezug auf ihre Aktivitäten in den Entwicklungsländern berücksichtigt.

In einem Zusatzverzeichnis werden allgemeinere Werke über die Internationalisierung der Schweizer Wirtschaft oder über für die Schweiz wichtige Wirtschaftsbereiche, ohne direkte Bezugnahme auf die Schweiz oder die Entwicklungsländer, aufgeführt. 


\section{Introduction}

Cet article a pour but d'offrir un survol de la littérature sur les effets des activités des firmes transnationales (FT) (1) suisses dans les pays en voie de développement. En introduction à la liste bibliographique des ouvrages et articles parus dans ce domaine de 1980 à juillet 1988, la première partie de l'article tente de relever les traits dominants de cette littérature et de la replacer dans le cadre des controverses qui ont surgi ces deux dernières décennies.

Le développement très rapide de la transnationalisation depuis les années soixante s'est accompagné d'une profusion d'ouvrages consacrés à l'explication de ce phénomène, à la dénonciation de certaines pratiques des firmes ou à la justification de l'apport de celles-ci dans la croissance et le développement économique. La Suisse n'est bien sûr pas restée à l'écart du débat sur les bienfaits et les méfaits des FT.

On peut distinguer plusieurs types d'études dans la littérature parue en Suisse:

1. Etudes partielles de certains apports des FT au développement ou dénonciations de certaines pratiques.

2. Etudes de certains secteurs d'activité importants pour les relations Suissepays en développement (industrie pharmaceutique par exemple).

3. Analyses coûts- avantages de certaines implantations précises de FT dans les pays en développement.

4. Contributions sur le développement de la théorie sur les investissements directs étrangers.

5. Contributions sur l'analyse de la structure interne des FT et de leur stratégie financière.

6. C'est dans le cadre du Programme national de recherche No 9 du Fonds national de la recherche scientifique que l'internationalisation de l'industrie suisse a été étudiée, avec les répercussions sur les structures de production de la Suisse. Le programme analysait notamment le processus d'industrialisation des nouveaux pays industrialisés, la concurrence dans le marché international, la compétitivité de l'industrie suisse (voir les ouvrages/ articles de Silvio Borner 1980, 1982, 1985, 1981 An: liste annexe) (2) et de F. Wehrle (1984).

Ce commentaire bibliographique fait référence surtout aux trois premiers types d'études. L'analyse des contributions plus théoriques ou des recherches sur la structure interne des firmes nécessiterait une référence à l'évolution des théories de l'investissement direct étranger. Par manque de place, nous n'approfon- 
dirons pas ces études pourtant importantes. Celles-ci sont néanmoins citées dans la bibliographie. Nous n'avons pas l'ambition de fournir des réponses précises sur l'effet globalement positif ou négatif des activités des firmes suisses, mais simplement de fournir quelques hypothèses sur certains effets et d'offrir un «panorama» général de la littérature.

\section{Investissements directs (3) suisses dans les pays en développement}

Une analyse de la répartition géographique des flux suisses de capitaux privés destinés aux pays en développement est rendue difficile par l'absence de statistiques précises des flux financiers par pays et par branches économiques.

De 1980 à 1983, en 1985 et 1986, plus de la moitié des investissements nets (4) suisses destinés aux pays en développement se sont dirigés vers l'Amérique latine et centrale (5). L'Afrique ne représentait qu'une part modeste (de $3,6 \%$ en 1981 à $14,4 \%$ en 1983, puis mouvements nets négatifs en 1984, 1985 et 1987).

Les investissements directs de la Suisse dans les pays en développement ne représentaient pourtant que le $7,6 \%$ des investissements directs totaux de la Suisse en 1985 (16,2\% en 1986), soit 849 millions de francs en 1985 sur une valeur totale investie à l'étranger de 11.236 millions de francs (Thomas Schlup, 1987, p.285).

En outre, les investissements directs suisses se dirigent surtout vers un nombre restreint de pays en développement (Argentine, Brésil, Mexique, Pérou, nouveaux pays industrialisés d'Asie, Turquie et Inde).

\section{Stratégie d'implantation des firmes transnationales dans les pays en développement}

Les formes d'internationalisation de la firme peuvent varier. La stratégie classique d'intégration verticale internationale ne semble pas avoir joué un rôle très important en Suisse: l'implantation de firmes d'extraction de matières premières pour assurer l'approvisionnement de la maison-mère est relativement rare pour les FT suisses (Alusuisse et les mines de bauxite par exemple) (6). La conquête des marchés ou la conservation de son accès sont sans doute les motivations primordiales pour la création d'une filiale d'une FT suisse dans un pays en développement (voir par exemple Gerster, Richard, 1987). La recherche de conditions de salaires avantageuses ne semble par contre pas être un élément très important. Très peu d'études examinent le rôle de la conclusion d'accords de protection des investissements, ou d'accords sur la double imposition, dans l'incitation des investisseurs (voir Gerster, Richard, 1987). 


\section{Nouvelles formes d'investissement}

Dès les années 70 , les FT cherchent à faire partager les risques de l'investissement par les partenaires des pays en développement. De «nouvelles formes d'investissement" vont se développer, en partie en réaction aux politiques restrictives de ces pays envers les FT. Les "joint ventures" permettent de partager le pouvoir avec des capitaux locaux ou l'Etat du pays hôte. Les "joint ventures" sont moins coûteux en capital et permettent, selon Peter Wuffli (1985, p.54), une meilleure intégration dans les pays hôtes. La participation locale au management améliore aussi l'accès aux marchés financiers et au marché du travail. Cette forme d'investissement offre en outre une protection accrue contre les nationalisations. Des problèmes peuvent tout de même surgir s'il y a des conflits avec le partenaire local. Le risque de diffusion non voulue de technologie est plus important. La création traditionnelle d'une filiale offre l'avantage d'un meilleur contrôle de la direction et de possibilités accrues de rapatriement de bénéfices.

Les accords de licences, la sous-traitance internationale et la coopération industrielle sont d'autres formes d'investissements pratiquées de plus en plus fréquemment par les firmes suisses. Une enquête auprès de 272 entreprises suisses a été menée à ce sujet dans le cadre du programme national de recherche No 9 (voir Borner Silvio, 1985 an: liste annexe).

\section{Traits dominants de la littérature}

II est indispensable de replacer la littérature existante dans le cadre des débats et des polémiques qui ont existé en Suisse ces 15 dernières années. La littérature est en effet fortement marquée par la dénonciation des activités de certaines FT.

La dénonciation de certaines pratiques publicitaires de grandes FT dans les pays en développement s'est intensifiée dans les années 70 . C'est sans doute l'affaire de lait en poudre de Nestlé qui a créé le plus de remous. L'ouvrage de Jean-Claude Buffle (1987) retrace le déroulement du boycott de Nestlé lancé aux Etats- Unis suite aux accusations portées contre la FT concernant sa politique publiciaire pour le lait en poudre dans le pays en développement. Le boycott a duré de 1977 à 1984. II avait été lancé par INFACT, coalition pour une action sur le lait pour nourrissons. Le procès de Nestlé contre le Groupe de travail Tiers Monde de Berne, après la publication "Nestlé tue les bébés" en 1974, constitue la première réaction importante d'une FT suisse face aux critiques. Nestlé, première FT suisse, est aussi la firme dont on parle le plus dans la littérature. L'adoption par l'OMS du code de commercialisation de substituts au lait maternel a entraîné une décrispation du débat. Pas pour longtemps d'ailleurs car des accusations de violations du code par Nestlé et la firme américaine American Home Products a provoqué le lancement, par des organismes de défense des consommateurs, d'une nouvelle campagne de boycott contre les produits de ces firmes (NZZ, 5.10.88). 
Plus du tiers des ouvrages et articles cités dans cette étude traitent de l'industrie chimique et pharmaceutique. C'est incontestablement la branche économique la plus étudiée. La nécessité ou non d'élaborer des codes de conduite internationaux pour mieux contrôler les activités des FT constitue aussi une préoccupation importante. Parmi les effets des activités des FT qui reviennent le plus souvent : l'emploi, les conditions de travail, les pratiques publicitaires controversées (lait en poudre et médicaments), les liens entre la politique du pays hôte et la FT. Ces aspects seront examinés plus en détail ci-dessous. Nous n'aborderons par contre pas le cas des transnationales bancaires, ni l'industrie d'armement.

\section{FT et coopération au développement}

Parmi les nouveaux thèmes abordés ces dernières années, citons les effets sur l'environnement et les liens entre les FT et la copération au développement. On reconnait actuellement que les dépenses faites au compte de la coopération au développement rapportent beaucoup à la Suisse, car sur chaque franc de la coopération, 94 centimes reviennent en Suisse par le biais de la vente de biens et de services (7). On connaît très peu le rôle que jouent les FT suisses dans ce domaine. Les biens et services financés dans le cadre de l'aide publique sont en effet attribués sur la base d'appels d'offres (voir Widmer et Walser, 1982; Levy, Ph, 1982 an: liste annexe) (8).

L'article de Klaus Leisinger (1986) montre la collaboration entre la DDA, I'EPFZ (Ecole polytechnique fédérale de Zürich) et la firme Ciba- Geigy dans un projet d'amélioration des rendements de la production de riz au Madagascar (projet «Taona Zina»). Comme la présentation de la plupart des projets de coopération, celle-ci ne présente que les effets positifs sur la formation, la production, ainsi que les effets sociaux et politiques.

\section{Secteurs ou activités négligés dans la littérature}

Malgré l'abondance de la littérature sur les FT suisses, beaucoup de branches économiques ou de thèmes restent peu étudiés. A part le secteur bancaire, les activités des FT des services restent dans l'ombre. Ce secteur va pourtant certainement prendre une ampleur considérable (9). Les activités des assurances suisses sont méconnues.

Si le grand public connaît Nestlé, Ciba-Geigy et Hoffmann la Roche, il ignorera l'existence d'une multitude de FT d'import- export et l'importante activité de la Société générale de surveillance, par exemple. Pourtant, cette dernière firme fait partie des 40 plus grandes entreprises suisses et occupe près de 20.000 personnes dans le monde. Cette firme occupe une position importante dans le domaine de la surveillance et du contrôle des transactions commerciales à l'échelle mondiale (attestation de qualité et de quantité, rapports d'inspection, 
contrôle des importations nationales, etc...)(10). Dans le secteur de l'électricité ou la construction, des études existent seulement sur Brown Boveri et Holderbank.

Plusieurs ouvrages étudient la stratégie des FT face aux critiques formulées contre elles (J.-C Buffle, 1986; P. Harrisson, 1983). La riposte des firmes a été plus ou moins heureuse selon les périodes : par exemple, discréditer les auteurs de critiques en disant que ceux-ci veulent porter atteinte au monde libre (J.-C. Buffle, p. 90). Autant les réseaux internes de circulation d'informations doivent être extrêmement bien développés entre filiale et maison- mère, autant l'information du public est restée souvent superficielle ou maladroite (liste simpliste de contre- arguments aux critiques, Medicaments et Tiers Monde, 1984, par exemple). Des efforts intéressants pour une information touchant un public plus large ont été entrepris par l'ancien Institut de recherche sur les multinationales (IRM), institut qui était financé par Nestlé (11).

\section{Effets des activités des FT suisse dans les PVD}

Une grande partie des études parues en Suisse relèvent les effets bénéfiques des activités des firmes suisses dans les pays en développement. Plusieurs études dénoncent certains abus dans ces mêmes activités. II existe pourtant encore peu d'études systématiques sur les effets positifs et négatifs de certaines activités. Examinons maintenant quelques aspects qui reviennent souvent dans la littérature, soit:

- l'apport des FT à la production nationale (7.1).

- la création d'emploi et la formation (7.2 à 7.4).

- l'apport technologique (7.5 et 7.6$)$.

\subsection{FT et son effet d'entrainement sur la production nationale du pays hôte}

La création d'une filiale dans un pays en développement contribue à l'augmentation du produit national du pays hôte. Cet apport de capital étranger permet, dans le domaine industriel ou agro-alimentaire, une transformation sur place et l'achat de matières premières et/ou de produits intermédiaires sur les marchés locaux. Cela peut contribuer au développement de l'exportation nationale de produits plus élaborés ou à la substitution aux importations. Les politiques de substitution aux importations de produits manufacturés ont été adoptées dans beaucoup de pays, avec pour corollaire la protection des producteurs locaux par des barrières douanières. Ces politiques ont incité les FT à poursuivre leur expansion grâce à l'ouverture de nouvelles usines ou par le rachat de firmes locales (Antoine Brawand, 1987 an). Selon les firmes, cet apport extérieur constitue un stimulant pour la concurrence nationale et permet ainsi une modernisation de l'économie. Pour certains auteurs, cela aurait permis aux FT de s'assurer une position dominante. Le cas de Brown Boveri au Brésil montrerait plutôt une 
élimination de la concurrence locale (voir Kurt Mirow, 1982).

D'autre part, l'effet net bénéfique de cet apport étranger sur la balance des paiements est contesté par certains auteurs (dont Pierre Harrisson, 1983). II faudrait en l'occurrence tenir compte du coût des importations de matières premières et de produits intermédiaires par la filiale, le coût de la présence de cadres expatriés et surtout du rapatriement des profits. La manipulation des prix de transferts entre maison- mère et filiale peut constituer un moyen efficace de rapatriement de fonds vers le pays siège ou vers un pays "paradis fiscal» (à ce propos: Brudon, P. 1983; Harrisson, P. 1983; November, A. 1981...).

\subsection{Création d'emplois}

La création d'emplois dans le pays hôte reste l'aspect le plus souvent cité pour souligner l'importance des activités des firmes suisses. Pour Felix Wehrle (1984), les 15 premières FT suisses employaient 100.370 personnes dans les pays en développement en 1980. La même année, ces 15 firmes employaient 160.640 personnes en Suisse et 383.970 dans les autres pays industrialisés. L'industrie alimentaire constitue, pour ces 15 firmes le principal employeur (46.000 places de travail dans les pays en développement), suivi par la chimie (27.000) et l'industrie des machines (18.000). Les filiales de toutes les FT ont employé entre 130.000 et 150.000 personnes dans les pays en développement, cela de 1980 à 1987 (12). La répartition entre les continents paraît relativement stable dans le temps, soit de $55 \%$ aux deux tiers des effectifs en Amérique latine. Voici la répartition en 1987 , pour un total de 142.543 emplois:

Répartition des effectifs du personnel employé par les entreprises suisses dans les pays en développement en 1987, par continents et en 8 du total.

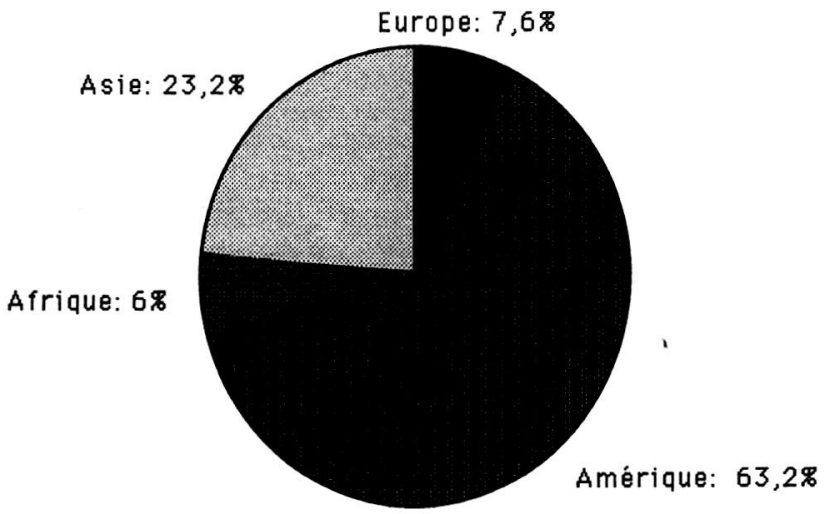

Source: graphique élaboré à partir du tableau 2.4.A.1. de la partie statistitique. 
L'implantation d'une filiale pouvant entraîner la disparition d'emplois traditionnels (industrie contre artisanat), il faudrait bien sûr connaître la création nette d'emplois. Quant au niveau de salaire, plusieurs études ont montré que dans l'ensemble, les FT paient des salaires plus élevés que les firmes locales ( $\mathrm{Ca}$ ves, Richard, 1982,p266). L'utilisation d'une technologie intense en capital peut aussi être une limite à la création d'emplois.

\subsection{Formation professionnelle}

L'apport de la FT à la formation professionnelle reste aussi un aspect important. La formation de cadres nationaux et le transfert de "know how", de savoir faire, peut représenter un apport appréciable pour des pays où les ressources pour l'éducation sont très limitées. L'expérience semble pourtant montrer que les industries s'installent avant tout dans les pays qui ont mis sur pied un système d'enseignement et de formation déjà assez développé (nouveaux pays industrialisés par exemple). Une formation générale du travailleur, «input» de l'entreprise, reste en effet importante pour cette dernière.

Il existe peu d'études systématiques de l'apport de la FT à la formation des travailleurs, même si beaucoup de publications de FT mentionnent cet aspect important. L'ouvrage de Hasler (1987) constitue une étude approfondie dans ce domaine.

Widmer et Walser (1982) étudient des projets de formation d'Holderbank, industrie du ciment:

«Pour produire du ciment, il ne suffit cependant pas d'acheter des installations industrielles demandant d'importants investissements en capitaux, puis de presser sur des boutons. La construction et l'exploitation de cimenteries impliquent un ensemble de connaissances et de savoir-faire étendus d'ordre technique, organisationnel et commercial, autrement dit l'existence de cadres, de spécialistes et d'ouvriers suffisamment qualifiés" (Widmer et Walser, 1982, p. 161-162). Pour ces auteurs, "le système de formation au sein des entreprises doit viser à élever le niveau des connaissances et du savoir-faire de tous les collaborateurs, afin que l'entreprise puisse, à long terme, fonctionner économiquement de façon autonome, c'est-à-dire sans dépendre de cadres et de spécialistes étrangers". Le personnel local doit être assisté à moyen terme par des spécialistes. Ces programmes de formation peuvent coûter chers (jusqu'à $5 \%$ de l'ensemble des frais d'implantation d'une usine). Les bénéfices pour l'entreprise sont multiples: éviter l'usure prématurée d'installations pour cause d'erreurs de service et d'entretien, éviter les pannes et une production en deçà de la capacité maximale. L'article de ces deux auteurs met en lumière une activité méconnue d'entreprises suisses: les contrats de formation et de gestion.

Lors de la création d'une nouvelle cimenterie en Equateur, par le gouvernement, et suite à un appel d'offres international, Holderbank a été choisie pour assurer la "conception et la mise en place de l'ensemble de l'organisation technique et administrative, le recrutement et la formation de l'ensemble du person- 
nel, et la direction technique de l'usine dans la phase de démarrage(...)" (Widmer et Walser, p.164). L'article décrit aussi l'appui d'Holderbank pour planifier, organiser et diriger pendant deux ans un centre de formation au Caire, projet financé dans le cadre du crédit mixte bilatéral Suisse- Egypte (voir aussi Gmünder et Jenny, 1988 pour les activités de formation professionnelle par Swisscontact en Indonésie).

Urs Hasler (1987) montre, sur la base de l'expérience de dix FT suisses en Amérique latine, que les efforts dans le domaine de la formation se concentrent surtout sur la préparation des expatriés suisses. Les buts exprimés de la formation sont notamment les suivants: assurer la relève de la gestion par le personnel local, augmenter la productivité, harmoniser la qualité des produits, améliorer l'image de marque dans le pays hôte et l'apport au développement dans ce pays.

\subsection{Conditions de travail}

Dans le domaine de l'emploi, beaucoup d'ouvrages et d'articles présentent cependant une situation moins réjouissante, surtout si l'on examine les conditions de travail et l'attitude des FT face aux syndicats. Si, comme nous l'avons vu, l'existence de bas salaires dans les pays en développement ne peut expliquer que très partiellement la localisation des FT suisses, les conditions de travail précaires et l'attitude envers les syndicats sont souvent dénoncées. Le manque de sécurité des travailleurs dans la manipulation de produits dangereux (voir le pesticide Galecron, 1982; Catrina, Werner, 1985 et Eternit, 1983 pour les dangers de l'amiante), la répression et les mesures antisyndicales dans certains pays (Harrisson, 1983: filiale Nestlé Perulac, République dominicaine, Colombie), jettent une autre lumière sur l'apport d'une FT. Ces pratiques confirmeraient la thèse selon laquelle les FT profitent de la fragmentation politique entre les pays, soit de réglementations différentes sur la sécurité des travailleurs et le temps de travail, des normes diverses sur la pollution, et d'une position affaiblie des organisations ouvrières dans certains pays.

\subsection{Transfert de technologie}

Le rôle très important des FT dans la "recherche et développement» et le transfert de technologie est relevé par beaucoup d'études. La croissance des pays en développement et la réduction de l'écart technologique entre le Nord et le Sud semble devoir passer par l'acquisition de technologies nouvelles qui sont développées surtout dans les pays industrialisés. L'examen du rôle des FT suisses dans certaines innovations technologiques (dans le domaine pharmaceutique ou des biotechnologies par exemple) mériterait à lui seul une étude bien plus longue que cet article. Le cas de l'industrie pharmaceutique a été largement étudié dans la littérature (par exemple dans: P. Brudon, 1983; Ciba-Geigy, 1985; November, 1981; Wenzel, 1986; Merciai, 1982 An).

Trois aspects sont souvent relevés dans la littérature: 
- Dépendance technologique envers les pays industrialisés.

La diffusion technologique par une FT semble constituer bien plus un transfert interne à l'entreprise (de la maison- mère à la filiale) qu'une véritable mise à disposition aux pays en développement d'une nouvelle technologie. Le pays d'origine reste bien souvent maître de l'innovation. En 1980, seul le $3 \%$ de la recherche des 15 plus grandes FT suisses a été exécutée dans les pays en développement (Gerster, 1987,p.)

- Adaptation de la technologie aux conditions locales.

Cette adaptation dépend de trois facteurs (R. Caves, 1982): la technologie utilisée par la FT est-elle adaptable à l'abondance de la main d'oeuvre? si oui, la FT va-t'elle l'adapter? la technologie est-elle plus adaptée que celle des firmes locales?

- Coût du transfert de technologie.

Le coût direct du transfert est aisément mesurable (paiement des redevances des brevets et licences). II en va pas de même pour le coût indirect: pratiques commerciales restrictives imposées par la maison-mère à sa filiale (restrictions aux exportations ou sur les sources d'approvisionnement des filiales), manipulation des prix de transfert...

\subsection{Brevets}

"L'innovation technologique est devenue un fruit défendu aux tiers: elle est propriété privée bien protégée, étant donné que chaque brevet déposé est entouré par un appareil juridique puissant. L'accès à la technologie de pointe est détenu par les grandes entreprises, qui la cèdent sous certaines conditions, en monnayant leur savoir" (November, 1981, p.189). Les implications des licences et brevets pour la Suisse et les pays en développement ont été examinés notamment par R. Gerster (1980). Le rôle de la Suisse est assez méconnu. Pourtant, selon cet auteur, $8,5 \%$ des patentes dans les pays en développement sont en mains de suisses ou de firmes suisses (Gerster, 1980, p.104), surtout dans l'industrie chimique et pharmaceutique. Pour cet auteur, les brevets accroissent la dépendance technologique des pays en développement alors que ceux-ci devraient justement pouvoir développer une capacité de recherche de technologies adaptées aux conditions locales.

\section{Etudes de cas d'implantations précises dans les pays en développement}

Les effets des activités des FT peuvent varier fortement selon la branche à laquelle appartient la FT. II existe encore peu d'analyses comparatives des stratégies des FT par branches. L'étude de Peter Wuffli (1985) sur la stratégie et les conséquences des différentes activités d'investissements de la Suisse au Mexique constitue un exemple précieux dans ce domaine. II analyse la stratégie et les caractéristiques des firmes suisses au Mexique par branches (industrie chi- 
mique, des machines, de la construction et industrie alimentaire).

Les FT utilisent souvent des études de cas précises pour illustrer leur apport au développement d'une région ou d'un pays (Ciba-Geigy et le Tiers Monde 1985; Nestlé, 1984 et 1985; Laya, J.-M., 1980; Rampini, 1980; Schneider, 1987). Ces études restent le plus souvent superficielles, car elles ne mettent souvent l'accent que sur les aspects positifs, comme d'ailleurs beaucoup d'autres ouvrages et articles d'opposants à certaines pratiques de FT qui ne mettent l'accent que sur certains aspects négatifs.

Pour être plus complet, certains auteurs recourrent à l'analyse coût- bénéfice. L'évaluation de tous les apports et les coûts monétaires et non monétaires d'une implantation d'une FT dans un environnement socio- économique et culturel reste difficile (problèmes de quantification de certains coûts et avantages).

Des études de cas approfondies existent pourtant:

Ciba-Geigy en Inde

L'ouvrage de Jürg A. Hauser et Walter $\mathrm{H}$. Rambousek évalue le rôle catalytique que l'industrie privée peut jouer dans une région de l'Inde. L'étude est consacrée aux effets positifs et négatifs de la filiale Ciba- Geigy à Goa, en Inde (production de pesticides). Les auteurs analysent l'intégration du projet dans l'environnement physique, social, économique, culturel et politique de l'Inde. Les principales conclusions sont les suivantes : les activités de Ciba-Geigy sont en accord avec plusieurs objectifs de développement de l'Inde. Les effets multiplicateurs jouent un rôle important grâce aux achats de matières premières et de produits intermédiaires en Inde. Les conditions d'emploi sont supérieures à la moyenne régionale. Les activités ont pour conséquence notamment une augmentation des inégalités régionales. Elles ne touchent pas les petits paysans. L'instrument d'évaluation utilisé, sorte d'analyse coût- avantage, permet, à l'aide d'une grille d'analyse élaborée, de confronter les buts de tous les différents acteurs impliqués avec les résultats obtenus. L'instrument permet une analyse intéressante sans passer par une quantification excessive.

Holderbank et INCSA- Costa-Rica.

L'analyse de Gerhard Schwarz (payée par la maison-mère Holderbank) de la fabrique de ciment INCSA au Costa Rica est un autre exemple d'étude d'impact de l'investissement direct (Schwarz, 1980). Pour préserver une image plus indépendante de l'analyse, elle a été menée avec la collaboration de Hans- Balz Peter, de l'Institut d'éthique sociale. Pour éviter une quantification excessive, l'auteur a élaboré un rapport social des effets de l'implantation. L'analyse répond à la question de savoir si la production de ciment au Costa-Rica répond à un besoin de base. L'auteur analyse ensuite l'effet sur la production locale, les effets sur la région (effets d'entraînement et importations induites),etc..

\section{Liens entre les Firmes transnationales (FT) et les Etats-nation}

Les différentes thèses sur les relations (conflictuelles ou non) entre la puissance économique d'une firme et la politique de l'Etat-nation marquent la plupart des 
études dans le domaine étudié ici.

Selon certains auteurs, l'influence des FT serait souvent surestimée car on confond taille et puissance. Les FT restent en fait, selon eux, soumises aux lois nationales. II est vrai que la simple comparaison entre le chiffre d'affaire d'une FT et le Produit national brut d'un petit pays en développement n'a pas beaucoup de sens. L'image véhiculée de efirmes monstres s'implantant dans les frêles Etats du Tiers Monde dans le seul but d'exploiter honteusement les populations" résiste mal à une analyse approfondie. Quelles que soient les critiques adressées à l'encontre des FT, beaucoup de pays en développement adoptent des mesures susceptibles d'encourager l'implantation de FT (dégrèvements fiscaux, investissements publics dans les infrastructures, création de zones franches, etc...). D'autre part, les FT occupent une place très importante dans le monde capitaliste et plus particulièrement dans certaines activités (dans la coopération au développement, ou les recherches dans les biotechnologies, par exemple).

Certains auteurs ont étudié de manière approfondie les liens entre la stratégie de la FT et l'environnement dans lequel il évolue, soit les contraintes que l'environnement économique, politico-juridique, sociologique et éducatif d'un Etat peuvent exercer sur le processus de management de la FT (Paul Michael Achleitner, 1985 an; Peter Schoop, 1983 an). Ces études relativisent l'image d'une firme toute puissante en mettant l'accent sur les contraintes. Reflets de la théorie classique, les écrits des FT postulent souvent l'absence de contradictions entre les buts des FT et la politique de développement du pays d'accueil. Plusieurs études sont consacrées à ce problème (les plus complètes: Baumer, J.-M et Gleich, A von, 1982; Hauser, Jürg et Rambousek, Walter H, 1984). J.-M. Baumer fait l'inventaire des objectifs des firmes et l'Etat colombien, pour analyser ensuite les relations (harmonie d'intérêt, conflit ou indifférence). Ainsi, par exemple, l'objectif de la firme de rapatriement du profit peut entrer en contradiction avec le désir du pays hôte de favoriser le réinvestissement sur place. Le but du profit maximal peut entrer en conflit avec celui de la protection de l'environnement, etc...

Nul ne contestera que la FT n'est pas une oeuvre de bienfaisance au service du développement des plus pauvres, mais que leurs activités induisent toute une série d'effets plus ou moins positifs pour le pays hôte. La FT, comme toute firme capitaliste, a pour exigence de survie à long terme la réalisation d'un profit. Cette firme défendra sa position économique dans ce but et poursuivra comme objectifs notamment la conquête de nouveaux marchés et la réduction du coût de production. La firme va redouter le contrôle des mouvements de capitaux, celui du rapatriement des bénéfices, l'instabilité politique, le système fiscal trop lourd, le risque de nationalisation et tous les autres facteurs limitant ses activités ou augmentant les risques de l'investissement.

La filiale de la FT peut aussi bénéficier d'avantages sur les entreprises locales, dont notamment:

- la faculté de déplacer plus facilement sa production dans des pays qui offrent des conditions plus avantageuses, ou pour le moins d'utiliser cette menace face 
aux revendications locales (de l'Etat ou surtout des travailleurs). Ceci réduit le pouvoir de négociation des Etats et des syndicats (Antoine Brawand, 1987).

- une capacité de management et de savoir-faire réunissant les expériences dans plusieurs pays, sur une longue période.

- un réseau d'information très développé entre la maison-mère et les filiales ou entre la FT et des centres de décisions économiques (bourses, marché des matières premières...)

- la possibilité de bénéficier d'une technologie de pointe, grâce aux dépenses importantes en Recherche et développement dans le pays d'origine.

A quel moment ces avantages économiques de la FT pourront-ils être utilisés pour éliminer la concurrence ou comme moyen de coercition envers l'Etat hôte?

Ce débat sur le pouvoir des FT mène à la question de l'opportunité ou non d'adopter des codes de conduite internationaux. En effet, si l'on considère que les FT possèdent un pouvoir abusif, la création de contre-pouvoirs permet de limiter cette évolution, soit par le renforcement du contrôle de l'Etat, l'organisation syndicale sur le plan international, l'organisation de la défense des consommateurs ou la réglementation internationale. Certains groupes de pression se forment au sein même de l'entreprise, tel que CANES, Convention d'actionnaires de Nestlé, par exemple. Sur le plan international, signalons le code OMS de commercialisation de substituts du lait maternel, le code sur les pratiques commerciales restrictives adopté par la CNUCED. Pourtant, actuellement, peu de volonté politique semble se dégager en faveur de nouveaux codes, dont le code général pour les FT (13). Le renforcement de ces divers contre-pouvoirs reste importante, ne serait-ce que par solidarité avec les populations qui subissent les conséquences d'un certain (dés)ordre économique international. Les conditions de travail mettant en péril la santé des employés, les pratiques publicitaires dans le but de vendre des produits inutiles dans le contexte socio-économique des pays en développement, voire dans certains cas dangereux pour les utilisateurs; tous ces aspects doivent être dénoncés avec force.

\section{Présentation de la liste bibliographique}

Compte-tenu de l'abondante littérature concernant les firmes transnationales, nous nous limitons dans cette bibliographie aux ouvrages et articles de revues spécialisées consacrés à l'étude des effets des activités des firmes transnationales suisses dans les pays en développement, de 1980 à mi1988. Nous ne considérons donc pas les effets sur l'économie suisse, ni les descriptions des FT sans référence à leurs activités dans les pays en développement.

Nous n'avons pas l'ambition d'avoir établi une liste exhaustive sur la question. S'il est relativement aisé de réunir une liste d'ouvrages parus en Suisse, il est beaucoup plus difficile de réunir tous les articles de revues, et plus encore les ouvrages parus à l'étranger qui traitent du même sujet. Pourtant, un flux d'informations du Sud au Nord serait extrêmement important, car la connaissance des études entreprises par les principaux intéressés dans les pays en développe- 
ment (ou les principales victimes!) serait primordiale. Les bibliographies présentées à la fin de certains ouvrages peuvent donner des indications sur ces études.

Une liste annexe mentionne les ouvrages ou articles plus généraux sur l'internationalisation de l'économie suisse ou sur des secteurs importants pour la Suisse, sans référence directe à ce pays ou aux pays en développement. Cette annexe mentionne aussi les ouvrages utilisés pour rédiger la première partie de cette contribution.

Une liste de tous les rapports annuels des FT serait trop longue. Ceux-ci peuvent pourtant contenir des informations précieuses sur leurs activités dans les pays en développement.

Toute une série de publications parlent régulièrement des effets des activités des FT suisses, par exemple:

- i3W. Entwicklungspolitischer Dokumentations- und Pressedienst. Bern

- Mosquito, Bern.

- Bulletin Nestlé, UITA, Union internationale des travailleurs de l'alimentation. Nouvelles syndicales, Genève.

- Vers un développement solidaire, Déclaration de Berne, Lausanne.

- Lettre d'information $\mathrm{CH}-6$, Fondation pour une 6ème suisse responsable, Trélex.

Les mots clefs utilisés pour caractériser le contenu des ouvrages sont les suivants:

- Effets des activités sur la balance des paiement.

- FT et concentration économique dans le pays hôte. Monopoles, oligopoles.

- Conditions de travail dans les filiales des FT, salaires, horaires de travail.

- Liens entre FT et coopération au développement.

- Effets des FT sur le développement régional dans le pays hôte.

- Emplois créés grâce à la FT.

- Liens entre la FT et l'Etat- nation, pouvoir politique des FT, objectifs de développement du pays et objectifs de la FT, législations nationales et FT.

- Influence des activités de la FT sur la concurrence, les firmes locales.

- Formation professionnelle au sein des FT.

- Localisation d'une filiale.

- Joint Venture.

- Organisations internationales et FT.

- Pouvoir et puissance économique de la firme.

- Pratiques publicitaires de la FT dans les pays en développement.

- prix et prix de transfert entre maison- mère et filiale.

- Stratégie financière et structure interne de la FT, relation entre la maison mère et sa filiale, management.

- FT et politiques de substitution des importations.

- Syndicalisme, relation filiale et syndicat, syndicats internationaux.

- transfert de technologie, patentes et brevets. 


\section{Notes}

1. La définition la plus large que l'on peut donner de la multinationale est une entreprise nationale contrôlant plusieurs filiales dans plusieurs pays (Encyclopédie Universalis, 1987). Parmi les multiples noms que l'on peut utiliser (firmes multinationales, internationales...), nour préférons le terme de firmes transnationales qui exprime mieux une certaine autonomie vis-à vis des Etats nationaux.

2. Les renvois à la bibliographie ou les sources sont simplement indiqués par le nom de l'auteur et l'année d'édition de l'ouvrage ou de l'article. La source complète se trouve dans la liste bibliographique, point 11, ou la liste annexe si l'on indique An.

3. Par investissement direct, on entend dans la statistique suisse un investissement dans une entreprise à l'étranger dans le but d'influencer durablement l'activité commerciale de cette dernière (T. Schlup, 1987).

4. Investissements nets= investissements bruts (y compris réinvestissements de bénéfices) moins rapatriements de capitaux (désinvestissement). Les rapatriements de bénéfices ne sont pas pris en compte.

5. D'après les tableaux 2.4.A.1. de la partie statistique des Annuaires Suisse-Tiers Monde 1981, 1984, 1987/88 et 1989.

6. Voir Campbell, Bonnie K pour les FT de l'aluminium (1983 an: liste annexe).

7. Voir l'Annuaire Suisse-Tiers Monde 1987/88, chap IV de la partie Revue.

8. Pour les mécanismes de passation des marchés, voir la brochure de Pierre Gygi.Suisse-Tiers Monde: Livraisons, prestations, investissements au titre de la coopération au développement.- Office suisse d'expansion commerciale, Zurich.

9. L'ouvrage de Schwamm et Merciai (1985) constitue une introduction à l'analyse des FT et les services.

10. Voir $\mathrm{CH}+6$. Lettre d'information No 10, janvier 1987, Trélex.- (Fondation pour une 6ème Suisse responsable).

11. Plusieurs livres édités par l'institut sont dans la liste annexe: Hamilton, G. 1984; Rigoni,R. 1985; Schwamm, H, 1985.

12. Voir les tableaux 2.4.A.2 de la partie statistique des Annuaires Suisse-Tiers Monde $1984,1987 / 88$ et 1989 . II faut tenir compte de l'approximation de ces chiffres, car les entreprises qui répondent aux enquêtes n'ont pas appliqué des règles uniformes pour la consolidation des effectifs.

13. Voir à ce propos le déroulement des négociations de la Commission des sociétés transnationales dans la partie Revue des Annuaires Suisse-Tiers Monde. 